\title{
Volumetric Overload Shocks in the Patho-Aetiology of the Transurethral Resection Prostatectomy (TURP) Syndrome and Acute Dilution Hyponatraemia: The Complete Evidence
}

\author{
Ghanem $\mathrm{AN}^{1 *}$, Ghanem SA${ }^{2}$, Ghanem $\mathrm{KA}^{3}$ and Nisha Pindoria ${ }^{4}$ \\ Retired Consultant Urologist, Mansoura University, Egypt
}

Received: December 27, 2017; Published: February 02, 2018

*Corresponding author: Ahmed N Ghanem, Retired Consultant Urologist, Mansoura University, No1 President Mubarak Street, Mansoura 35511, Egypt, Tel: 001020883243/0020502267028; Email: anmganem1@gmail.com

\begin{abstract}
Objective: To present the complete evidence that volumetric overload shocks (VOS) is the real patho-aetiology of the transurethral resection of the prostate (TURP) syndrome and acute dilution hyponatraemia as based on two clinical studies plus physical and physiological studies that prove Starling's law is wrong and provide an alternative mechanism.

Patients and methods: Cases Series of 23 patients divided into 3 groups. Group 1 of 3 patients were mistaken for one of the recognised shocks and treated with volume expansion. Group 2 of 10 patients were correctly diagnosed as volumetric overload shock and treated with hypertonic sodium. Group 3 of 10 patients were encountered in the prospective study and randomised between treatments. A physical study on the hydrodynamics of the porous orifice $(G)$ tube and a physiological study on the hind limb of a sheep were conducted.

Results: The first 3 patients of 23 TURP syndromes died as they were mistaken for one of the recognised shocks and treated with volume expansion. The remaining 20 patients were correctly diagnosed as VOS and treated with hypertonic sodium therapy (HST) and all survived. The physical study revealed the hydrodynamics of the porous orifice (G) tube providing a replacement for Starling's law. The physiological study further confirms these findings.

Conclusion: Diagnosing TURP syndrome mistakenly for one of the recognised shocks and treating with volume expansion caused death. Correctly diagnosing TRRP syndrome as VOS and treating with HST caused survival of all patients. Based on physical and physiological studies evidence that prove Starling's law is wrong and provide an alternative mechanism is presented.

Keywords: Hyponatraemia; Shock; The Transurethral Prostatectomy (TURP) Syndrome; The Adult respiratory Distress Syndrome (ARDS), Starling's law, Capillary hydrodynamics

Abbreviations: VOS: Volumetric Overload Shocks; VOS1: Volumetric Overload Shock, Type 1; VOS2: Volumetric Overload Shock, Type2; TURP: The Transurethral Prostatectomy; ARDS: The Adult Respiratory Distress Syndrome; MVOD: The Multiple Vital Organ Dysfunction/Failure Syndrome; HN: Hyponatraemia; HST: Hypertonic Sodium Therapy; G Tube: The Porous Orifice Tube
\end{abstract}

\section{Definitions}

The transurethral resection of the prostate (TURP) syndrome is a severe vascular hypotension reaction that complicates endoscopic surgery as a result of massive irrigating fluid absorption causing severe acute dilution hyponatraemia (HN) of $<120 \mathrm{~m} \mathrm{~mol} / \mathrm{l}$ [1]. Volumetric Overload Shock (VOS) is a condition caused by massive fluid infusions and is of two types; Type one (VOS1) and Type two (VOS2). VOS1 is induced by sodium-free fluid gain such as $1.5 \%$ Glycine used as irrigating fluid during endoscopic surgery such as TURP [1]. It has been reported with other fluids such as Glucose, Mannitol and Sorbitol. It is known as TURS or HN shock [2] as HN is a marked serological marker for the condition [3]. VOS2 is induced by massive infusion of sodium-based fluids such as normal saline, Ringer, Hartmann, plasma and plasma substitutes and/or blood transfusions that may complicate the therapy of VOS1. VOS2 also complicates fluid therapy in critically ill patients suffering from other known shocks such as trauma, hypovolaemic, haemorrhagic and septicaemic shocks and presents with the multiple vital organs dysfunction (MVOD) or failure syndrome. The adult respiratory distress syndrome (ARDS) [4] is another name under which VOS2 is reported. Both VOS1 and VOS2 are iatrogenic complications of fluid therapy and occur only in hospitals.

\section{Introduction}

The clinical studies demonstrate that the transurethral resection of the prostate (TURP) syndrome presents as 
volumetric overload shock (VOS). Mistaking VOS for one of the recognised shocks and treating it by volume expansion causes death of the 3 patients. Correctly diagnosing VOS and treating with hypertonic Sodium therapy (HST) saved the lives of all 20 cases. We further investigated Starling's law which underlies the principles of fluid therapy proving it wrong and provided the replacement mechanism using physical and physiological studies.

Why should TURS be recognized as VOS? As shown here VOS1 is the real patho-aetiology of TURS which has HN as a clear serological marker. This makes it easier to recognize VOS2 which unlike VOS1 has no clear serological marker. It also helps to establish the correct and life saving therapy of hypertonic $5 \% \mathrm{NaCl}$ or $8.4 \%$ Sodium Bicarbonate. It has also helped in realizing that the physiological law of Starling, which underlies the principles of fluid therapy in clinical practice, is in fact incorrect. From the literature review it will be realized that TURP syndrome presents as vascular hypotension shock to the anaesthetists and surgeons during the surgery that should not be mistaken for one of the recognized shocks. By next morning it presents as HN coma to physicians. VOS1 has been induced in animals under clean experimental conditions in the absence of hemorrhage and sepsis [5].

\section{Literature Review}

TURP syndrome was first reported by Creevy [6] as acute water intoxication when distilled water was used as irrigating fluid for TURP. Water intoxication caused intravascular red cell haemolysis and acute renal failure. Shift to osmotic solutions was made and $1.5 \%$ Glycine gained popularity. Harrison et al. [2] reported TURP syndrome as acute dilutional hyponatraemic shock after massive gain of Glycine irrigant. However, TURP syndrome is not limited to TURP. It may affect any endoscopic surgery and has been reported in women undergoing Transcervical Endometrial Resection [7,8]. It may also affect women undergoing any surgery following excessive 5\% Glucose infusions [3]. TURP syndrome manifests as shock during surgery and by next morning it manifests as HN encephalopathy coma [9]. TURS may be mistaken for other recognized shocks such as septicaemic [10], hemorrhagic [11-13] and cardiogenic $[14,15]$ shock. VOS 2 may complicate all types of shocks during fluid therapy and the transition is seamless and hard to detect. It may be called the irreversible shock. The only way to detect VOS 2 is the sudden acute increase in body weight or accurate fluid balance during resuscitation. The serum solutes changes particularly HN have been reported by all authors [16-18].

TURS may present as HN encephalopathy coma [3,7-9], cardiogeic shock or cardiac arrest [16], respiratory failure or arrest [19] and acute renal failure among other vital organs involved. Visual loss has also been reported [20]. Post-mortem examination has been documented [21]. TURP syndrome has been attributed to Glycine and ammonia toxicity [22] but it has also been reported with Mannitol [22] and Glucose [23].
Professor Hahn et al reported 480 articles of which $>340$ articles are on TURP syndrome [PubMed search December 2016] investigating the fluid and electrolytes dynamics [24], effect of over hydration on cardiac muscle [25] and other tissues [26], effect on renal function [27] and compared Glycine to Mannitol [28]. Professor Hahn favoured the toxicity of Glycine as the patho-aetiological cause of TURS. Ghanem and Ward introduced the concept of volumetric overload in the patho-etiology of TURS [1]. Ghanem confirmed the effectiveness of hypertonic $5 \% \mathrm{NaCl}$ or $8.4 \%$ Sodium Bicarbonate both as anecdotal evidence [29] and in a prospective study [1] and also investigated the underlying faulty physiological law of Starling for the capillary interstitial fluid transfer $[30,31]$. Ghanem went further to prove that VOS is the real patho-aetiology of the TURP syndrome and Starling law is wrong [31-34].

\section{Patients and Methods}

This article is based on 4 different studies, two clinical of which one is based on 23 cases series and another is a prospective study, plus physical and physiological studies that prove Starling's law for the capillary-interstitial fluid transfer is wrong. We report 23 case series divided into 3 groups of patients. Group 1 of 3 patients were treated as one of the known shocks by conservative treatment (CT) of volume expansion and all died. Group 2 of 10 patients were treated as volumetric overload shock (VOS) with HST of $5 \% \mathrm{NaCl}$ or $8.4 \%$ Sodium Bicarbonate and all survived. Group 3 were 10 symptomatic patients encountered during a prospective study on 100 patients [1] and were randomised between CT and HST; 5 patients in each group named group 3.1 and 3.2, respectively. Accurate data recording on each patient included age, body weight and volumetric fluid balance during the operation, immediate pre and postoperative times. Serum solute concentration changes were also recorded at pre and post operative times.

A prospective study of 100 consecutive patients undergoing TURP was carried out with the approval o the Medical Ethical Committee. A standard procedure was performed, using an irrigating resectoscope (Stors), 1.5\% Glycine irrgant (at a height of $80 \mathrm{~cm}$ above the heart) and suction drainage (Haemonetics Cell Saver IV), which measured blood loss. The absorbed volume of $1.5 \%$ Glycine was the difference between volume used and returned. Bumetanide $1 \mathrm{mg}$ was given at the end of the procedure. The volume and type of per-and 24-h post-operative intravenous fluids infused were recorded. Pre-and post-operative urinary cultures were performed on all patients and blood culture was performed on those showing signs of post-operative circulatory shock. Blood electrolytes, serum osmolality, Glycine, Serin and Alanine amino acids were measured on admission to the hospital (A), after anaesthetic induction (B), on termination of the procedure $(\mathrm{C})$ and on the first post-operative morning (D). Further measurements were carried out on symptomatic patients, who were randomised between HTS and conservative treatment. Known types of shocks were excluded by quantifying blood loss and doing blood cultures. 


\section{Open Access Journal of Surgery}

\section{Results}

The reported cases demonstrate that the volumetric overload (VO) is responsible for the induction of dilution hyponatraemia (HN) and TURP syndrome. A summary of VO causing both types of VOS1 and VOS2 is sown in (Figure 1 \& Table 1) shows the summary and comparing data on the 3 groups of patients. (Figure 2) shows the drop in serum sodium concentration (HN) and osmolality; characterising VOS1. The volume inducing VOS1 is 3.5-5 litres. The volume inducing VOS2 is twice to three times as much as shown in (Figure 1) in litres and as \% body weight. Mistaking VOS for a recognized sock and treating it with further volume expansion is lethal. While using HST of $5 \% \mathrm{NaCl}$ or $8.4 \%$ Sodium Bicarbonate is lifesaving. The HTS therapy induces massive diuresis and full recovery. This is proved by both case series as well as the prospective randomised trial on the 100 TURP patients [1] among whom the 10 symptomatic cases of Group 3 were randomised between the therapies.

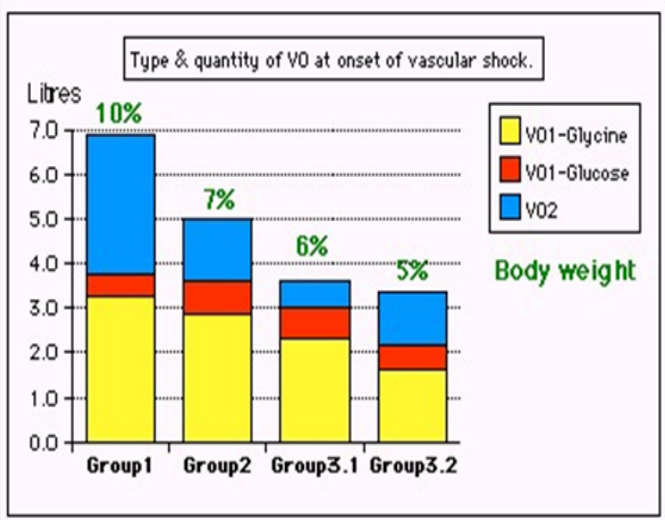

Figure 1: Shows the type (Sodium-free fluid VO1 and sodium based fluid VO2) and means quantity of fluid in litres and as percent of body weight at the time of occurrence of volumetric overload shock (VOS).

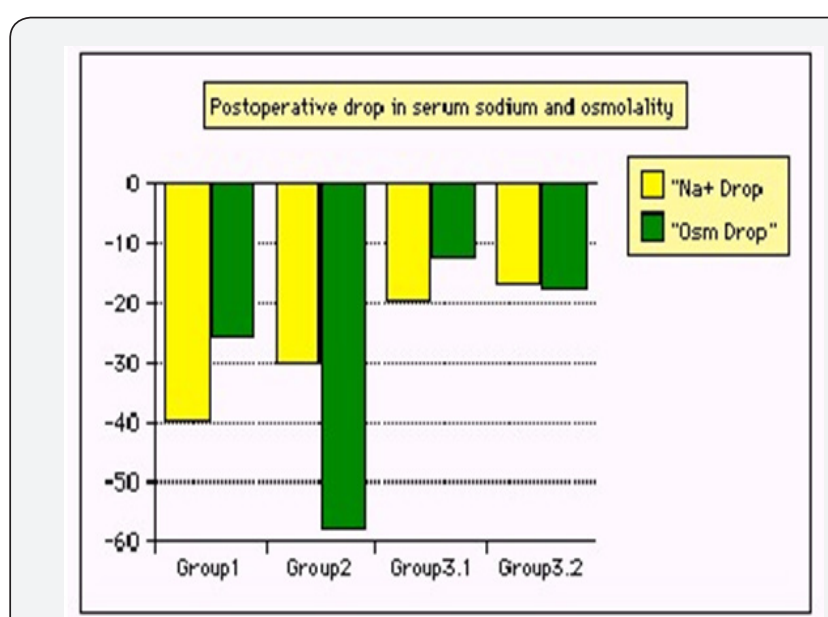

Figure 2: Shows the mean postoperative drop in serum sodium and osmolality in the 3 patients groups.

Table 1: Shows the mean summary of data, therapy and outcome comparing the 3 groups of patients.

\begin{tabular}{|c|c|c|c|c|c|c|c|}
\hline A & B & C & D & $\mathbf{E}$ & $\mathbf{F}$ & G & H \\
\hline & Gr 1 & Gr 2 & Gr 3 & Gr 3.1 & Gr 3.2 & Normal & Units \\
\hline Number of Patients & 3 & 10 & 10 & 5 & 5 & Mean & \\
\hline Age & 71 & 70 & 75 & 72 & 78 & 72 & Year \\
\hline Body Weight (BM) & 69 & 70 & 68 & 71 & 65 & 69 & $\mathrm{Kg}$ \\
\hline \multicolumn{7}{|c|}{ Postoperative serum solute concentration } & Preoperative \\
\hline Osmolality & 271 & 374 & 276 & 282 & 271 & \multicolumn{2}{|c|}{$292 \mathrm{~m} \mathrm{~mol} / \mathrm{l}$} \\
\hline $\mathrm{Na}+$ & 110 & 108 & 120 & 119 & 121 & \multicolumn{2}{|c|}{$139 \mathrm{~m} \mathrm{~mol} / \mathrm{l}$} \\
\hline $\mathrm{Ca}++$ & 1.69 & 1.79 & 1.85 & 1.84 & 1.86 & \multicolumn{2}{|c|}{$2.22 \mathrm{~m} \mathrm{~mol} / \mathrm{l}$} \\
\hline $\mathrm{K}+(\mathrm{P}<05)$ & 5.6 & 4.8 & 5 & 4.9 & 5 & \multicolumn{2}{|c|}{$4.46 \mathrm{~m} \mathrm{~mol} / \mathrm{l}$} \\
\hline $\mathrm{Co}_{2}(\mathrm{P}=.002)$ & 23 & 23 & 25.5 & 24 & 26.4 & \multicolumn{2}{|c|}{$27.3 \mathrm{~m} \mathrm{~mol} / \mathrm{l}$} \\
\hline Glucose & 13.2 & 17.3 & 16.4 & 15.9 & 16.9 & \multicolumn{2}{|c|}{$6.2 \mathrm{~m} \mathrm{~mol} / \mathrm{l}$} \\
\hline $\operatorname{Urea}(\mathrm{P}=.0726)$ & 26.5 & 9 & 6.6 & 6.8 & 6.4 & \multicolumn{2}{|c|}{$6.7 \mathrm{~m} \mathrm{~mol} / \mathrm{l}$} \\
\hline Bilirubin $(\mathrm{P}<.05)$ & 19 & 16 & 8 & 6 & 9 & \multicolumn{2}{|c|}{$7 \mathrm{~m} \mathrm{~mol} / \mathrm{l}$} \\
\hline AST & 124 & 32 & 20 & 18 & 21 & \multicolumn{2}{|c|}{$20 \mathrm{~m} \mathrm{~mol} / \mathrm{l}$} \\
\hline Protein & 43 & 52 & 48 & 44 & 52 & \multicolumn{2}{|c|}{$62 \mathrm{~m} \mathrm{~mol} / \mathrm{l}$} \\
\hline Albumin & 18.9 & 16.2 & 7.5 & 7.8 & 7.2 & \multicolumn{2}{|c|}{$8 \mathrm{~m} \mathrm{~mol} / \mathrm{l}$} \\
\hline $\mathrm{Hb}(\mathrm{P}=.0018)$ & 119.3 & 127.9 & 114.5 & 105.2 & 123.8 & \multicolumn{2}{|c|}{$138.8 \mathrm{~m} \mathrm{~mol} / \mathrm{l}$} \\
\hline WCC $(\mathrm{P}<.005)$ & 18.9 & 16.2 & 7.5 & 7.8 & 7.2 & \multicolumn{2}{|c|}{8.0 per HFP } \\
\hline Glycine & \multicolumn{5}{|c|}{10499} & \multirow{3}{*}{\multicolumn{2}{|c|}{$293 \mu \mathrm{mol} / \mathrm{l}$}} \\
\hline Therapy & CT & HST & Random & HST & CT @ & & \\
\hline Outcome & \multicolumn{3}{|c|}{ Death Full Rec } & Full Rec & Morb.@ & & \\
\hline
\end{tabular}


Of the prospective study, the mean age of the patients was 74 years $(\mathrm{SD} \pm 4)$, weight was $70.8 \mathrm{~kg}( \pm 8.6)$, weight of resected prostatic tissue was 30.8gr $( \pm 21.7)$ and resection time was $56.5 \mathrm{~min}( \pm 27.3)$. The mean volume of $1.5 \%$ glycine used for irrigation per procedure was 16.73 litres $( \pm 10.38)$. The mean per-operative volume of glycine absorbed, total fluid gained and blood loss were $0.6( \pm 0.7), 1.57( \pm 0.98)$ and $0.356( \pm 0.148)$ litres respectively. The mean and SD of volumetric overload of symptomatic patients are show in (Figure 3).

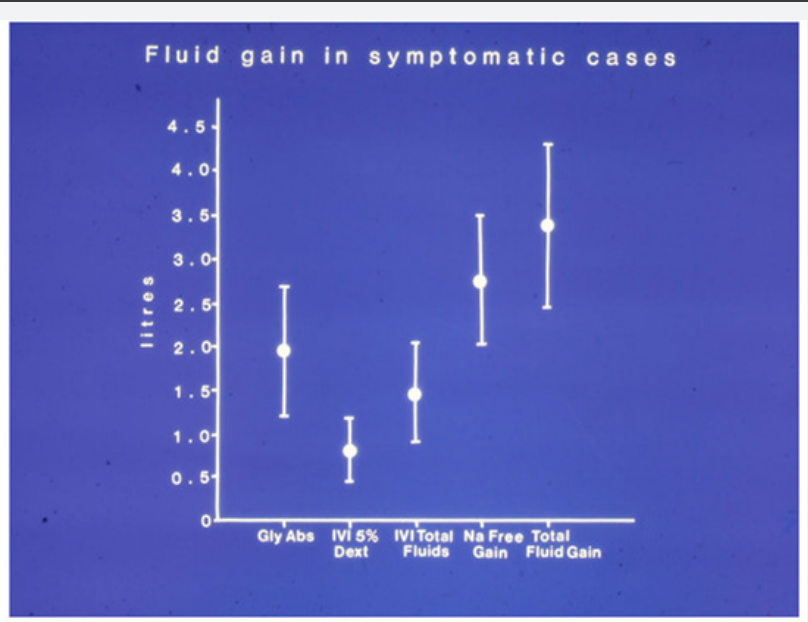

Figure 3: The means and standard deviations in symptomatic patients of Glycine absorbed (Gly abs), intravenously infused $5 \%$ Dextrose (IVI Dext) Total IVI fluids, Total Sodium-free fluid gained (Na Free Gain) and total fluid gain in litres (I).

A total of 20,10 and $4 \%$ of all patients showed a drop in post-operative serum sodium concentration of $>10,>15$ and $>20 \mathrm{mmol} / \mathrm{l}$, respectively. A significant relationship existed between the volume of glycine absorbed and the post-operative changes in serum glycine and sodium concentrations $(\mathrm{P}=0.0001)$. A significant relationship between the post-operative drop in serum sodium concentration and total volumetric gain, including IVI fluids, was also observed ( $\mathrm{P}=0.0001)$.

\section{Treatment}

Five of the 10 patients who showed signs of the TURP syndrome were treated with $\mathrm{HST} 5 \% \mathrm{NaCl}$ infused at a rate of $200 \mathrm{ml} / 20$ minutes. The other 5 patients plus the 2 patients with asymptomatic drop in serum sodium of $16 \mathrm{mmol} / \mathrm{l}$ were treated conservatively (CT). In the conservatively treated group, 2 patients appeared to suffer hypovolaemic shock despite volumetric overload and they all had the criteria of the TURP syndrome, they were treated with "guarded" volume expansion. The first patient received 1 unit of blood and 1 litre of Haemaccel with bumetanide $2 \mathrm{mg}$, atropine and aminophylline; the second patient received 1.5 litre of Haemaccel, atropine and a further dose of bumetanide. The 2 patients with asymptomatic hyponatraemia were each given a further dose of diuretic.

The changes in serum sodium and osmolality of patients treated with hypertonic sodium and those treated conservatively were compared. Those treated with hypertonic sodium responded promptly and recovered fully passing between 2.5 and 4.5 litres of urine. Serum sodium concentration was elevated by the end of infusion to $132.5 \mathrm{~m} \mathrm{~mol} / \mathrm{l}( \pm 0.2)$ and had returned to normal level by next morning. No complications or residual signs were observed in this group. One patient of the conservatively treated group developed cerebro-vascular accident with hemiplegia and fully recovered with a delayed infusion HhST.

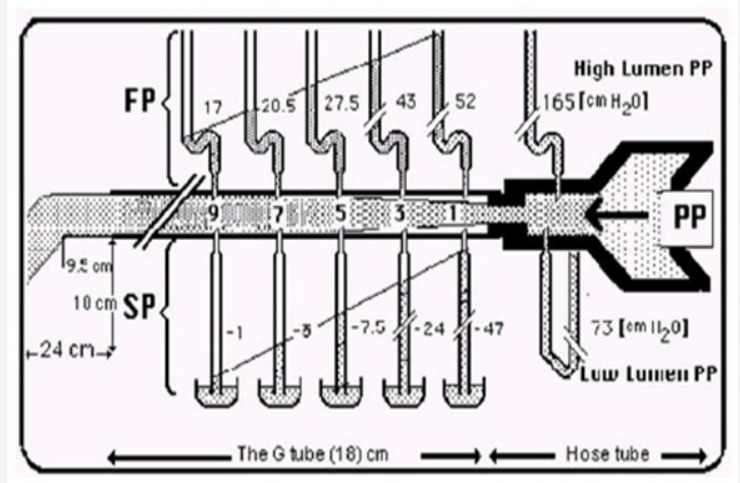

Figure 4: Shows the lumen pressure (LP) components of flow pressure (FP) and side pressure (SP) of a rubber orifice tube as measured by manometers with needles inserted at various $\mathrm{cm}$ distances from the inlet. When the needle's bevel faces upstream it measures FP (Top manometers) and when acing downstream it measures SP (Bottom manometers). 
The hydrodynamics of a rubber inlet tube that demonstrates the negative side pressure (SP) gradient exerted on its wall as well as the flow pressure (FP) components of its lumen pressure (LP) is shown in (Figure 4). The hydrodynamics of the $\mathrm{G}$ tube is shown in (Figure 5). The G-C phenomenon is shown in (Figure 6). It is observed from the presented results that the hydrodynamics of the $\mathrm{G}$ tube is totally different from Poiseuille's tube. The orifice of the $\mathrm{G}$ tube creates a negative side pressure gradient on its wall inducing a suction force which is transmitted to the surrounding chamber $\mathrm{C}$ creating a dynamic magnetic field like fluid G-C circulation that rapidly irrigates the $\mathrm{C}$. The orifice thus transfers the PP from a filtration force in Poiseuille's tube into a suction force in the G tube.

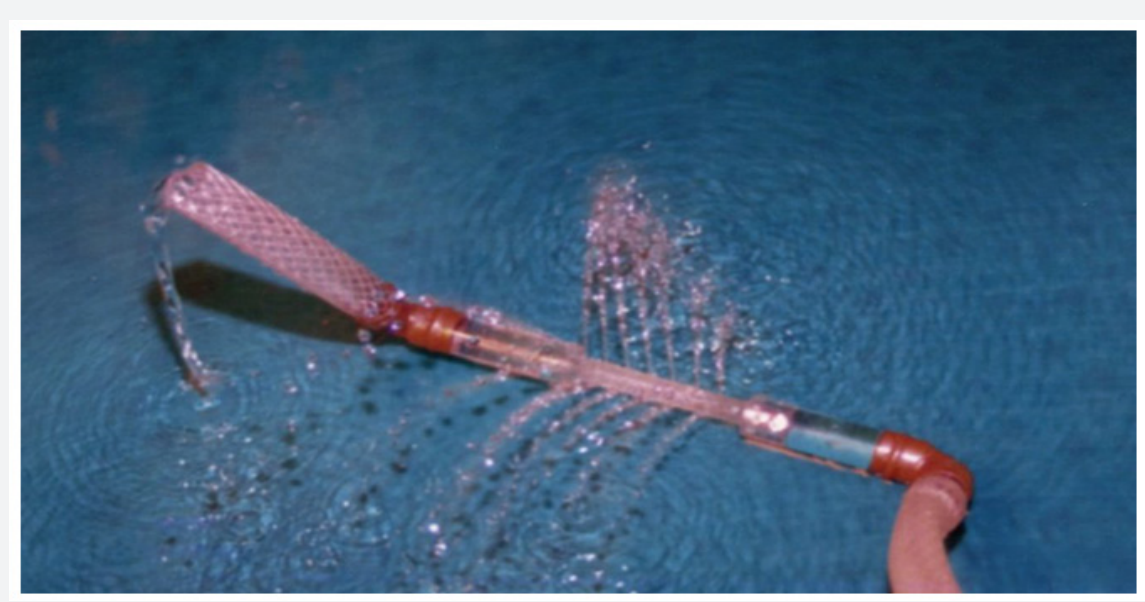

Figure 5: Shows the hydrodynamic of a porous oriice $(\mathrm{G})$ tube. The side pressure (SP) gradient exerted on its wall turns from negative near the inlet to positive near the exit. The magnetic field like G-C circulation is shown when the tube is placed in a surrounding chamber but can be seen at the top of the photo.

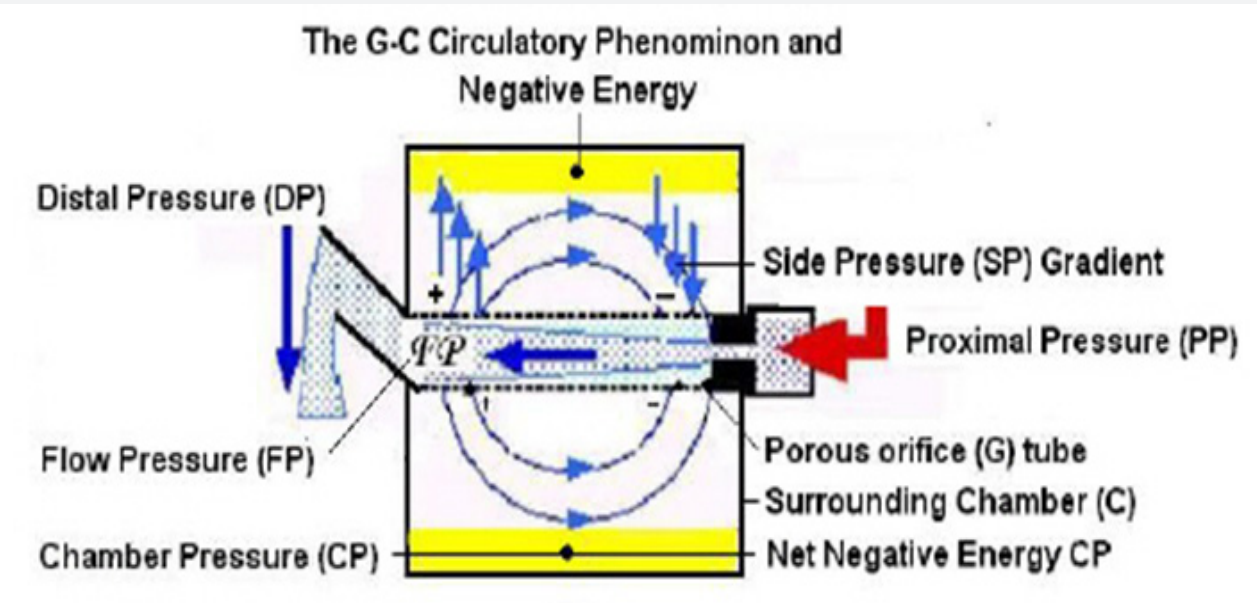

Figure 6: Shows a diagram of the G-C circulation based on several photographs creating a net negative pressure in $\mathrm{C}$ (Highlighted in yellow).

Increasing the PP enhances the G-C circulation while reducing it slows it down. The distal pressure has the opposite effect. Increasing the DP slows down the G-C circulation and turns the pressure in $\mathrm{C}$ into positive with increased volume. The effect of increasing the orifice diameter has $U$ or inverted Bell shaped effect on SP and CP. The G-C circulation thus offers a complete and correct replacement for Starling's law. In the physiological experiments running the fluid through the artery made the capillary act like a G tube causing suction through its proximal part and filtration through the distal part inducing a rapid circulation between the capillary lumen and surrounding ISF space with net negative pressure in the ISF space i.e. without oedema formation. Reversing the circulation and making fluid to run through the vein caused positive SP with filtration causing massive oedema with increased weight of the limb. Changing the circulating fluid from Normal Saline to Plasma Substitute (Haeemaccel) made no difference on the limb during both arterial and venous circulations.

The absence of oncotic pressure on using Normal Saline does not cause oedema. Reversing the inflow from venous back to arterial caused absorption of all the accumulated fluid in the ISF space and restored its negative pressure. This is a direct physiological proof that Starling's Law is wrong and the 
hydrodynamics of the $\mathrm{G}$ tube provide the correct replacement. The presented studies complement one another and provide the complete evidence on the new VOS causing the TURP syndrome and acute hyponatraemia.

\section{Discussion}

The presented evidence from the case series demonstrates that sodium-free fluid of $1.5 \%$ Glycine and 5\% Glucose induces VOS1 causing dilution of all serum solutes concentration of which $\mathrm{HN}$ is the most marked. It presents with shock during surgery and by next day it becomes HN encephalopathy coma. But, all vital organs are affected with dysfunction or failure due to congestion and oedema. Thus the clinical picture of VOS1 is coma, convulsion, disrythmia, annuria, respiratory distress, liver dysfunction, haematological disorder and paralytic ileus plus oedema or anasarca. The volume of gained fluids inducing VOS1 and VOS2 is shown in (Figure 1). So, these patients though in shock are not hypovolaemic but rather hypervolaemic as evidenced by the dilution of PV and ISF solute contents.

The results of the prospective study have shown that the TURP syndrome is precipitated by sodium-free fluid volumetric overload, the result of Glycine absorption and intravenously infused fluids. The measured mean volume of Glycine absorption, blood loss and changes of serum electrolytes are similar to those reported by other authors [9-12]. Data indicate that a volume of $>2$ litres, gained in one hour, can lead to the TURP syndrome; >3.5 litres precipitates VOS1 and multiple system dysfunctions (Figure 2). No patient with the syndrome had a blood loss exceeding 0.4 litres; unlike hypovolaemic and septicaemic shock, $5 \% \mathrm{NaCl}$ was shown to be the treatment of choice as further isotonic fluid and blood infusion were contraindicated. The G-C circulation phenomenon causes rapid mixing of fluids in the lumen of the $G$ tube and a surrounding fluid chamber compartment C. Its efficiency in exchanging materials between the $\mathrm{G}$ tube and chamber $\mathrm{C}$, akin to 'capillary and ISF compartments' is remarkable.

The shape of pores, whether hole or slit makes no difference. Large particles trapped in chamber $\mathrm{C}$ require regular cleaning. The negative energy of the $\mathrm{G}-\mathrm{C}$ circulation is a considerable force. This negative energy force effectively irrigates chamber $\mathrm{C}$, allowing rapid transfer of fluid and particles into and from chamber $\mathrm{C}$, akin to the passage of fluids with 'oxygen and nutrients including protein molecules into the ISF space and washing out waste products while preventing excess ISF accumulation. Guyton and Coleman's [35] capsule provided excellent evidence on the negative energy pressure of the ISF induced by the dynamic capillary flow, as demonstrated by the net negative $\mathrm{CP}$ in the G-C apparatus (Figures 6).

The presented evidence demonstrates that the capillaries act like $\mathrm{G}$ tubes in every aspect of the experiments. It allows rapid capillary- ISF transfer inducing a net negative pressure in the ISF space which prevents fluid accumulation in ISF space and oedema formation. On the other hand making capillary act as Poiseuille's tube by reversing the flow through the vein caused massive oedema formation and positive ISF pressure with matching increase in the limb's weight.

The presented complete evidence demonstrates that VOS is the real patho-aetiology of the TURP syndrome and acute hyponatraemia. Treating this condition by mistaking it for one of the recognised shocks by further volume expansion is lethal. Using hypertonic $5 \% \mathrm{NaCl}$ or $8.4 \mathrm{NaCo} 3$ is curative. The physical evidence demonstrate that Starling's law for the capillary interstitial fluid flow is wrong and provide the replacement mechanism of the $G$ tube. The physiological evidence confirmed that the capillary works as a $\mathrm{G}$ tube.

The literature review demonstrated that the TURP syndrome presents as shock that is usually mistaken for one of the recognized shocks and treated with volume expansion causing death. TURP syndrome was first reported by Creevy [6] as acute water intoxication when distilled water was used as irrigating fluid for TURP. Shift to osmotic solutions was made and $1.5 \%$ Glycine gained popularity. Harrison et al. [2] reported TURP syndrome as acute dilutional hyponatraemic shock after massive gain of Glycine irrigant. TURP syndrome manifests as shock during surgery and by next morning it manifests as HN encephalopathy coma [9]. TURS may be mistaken for other recognized shocks such as septicaemic [10], hemorrhagic [11-13] and cardiogenic $[14,15]$ shock. VOS 2 may complicate all types of shocks during fluid therapy and the transition is seamless and hard to detect. The serum solutes changes particularly $\mathrm{HN}$ have been reported by all authors [16-18].

TURS may present as HN encephalopathy coma [3,7-9], cardiogeic shock or cardiac arrest [16], respiratory failure or arrest [19] and acute renal failure among other vital organs involved. Visual loss has also been reported [20]. Post-mortem examination has been documented [21]. TURP syndrome has been attributed to Glycine and ammonia toxicity [22] but it has also been reported with Mannitol [22] and Glucose [3]. Professor Hahn et al. [24] reported 480 articles of which $>340$ articles are on TURP syndrome [PubMed search December 2016] investigating the fluid and electrolytes dynamics [24], effect of over hydration on cardiac muscle [25] and other tissues [26], effect on renal function [27] and compared Glycine to Mannitol [28]. Professor Hahn favoured the toxicity of Glycine as the patho-aetiological cause of TURS. Ghanem and Ward introduced the concept of volumetric overload in the patho-etiology of TURS [1]. Ghanem confirmed the effectiveness of hypertonic $5 \% \mathrm{NaCl}$ or $8.4 \%$ Sodium Bicarbonate both as anecdotal evidence [29] and in a prospective study [1] and also investigated the underlying faulty physiological law of Starling for the capillary interstitial fluid transfer $[30,31]$. Ghanem went further to prove that VOS is the real patho-aetiology of the TURP syndrome and Starling law is wrong [31-34]. This investigation started in 1985 and concluded in 2017 by the discovery of two new types of shock and a replacement for a faulty physiological law. 
It started with the observation that during resuscitation of shock of the TURP syndrome the patient died and post mortem examination revealed gross oedema of all organs and tissues. The occurrence of gross oedema with severe hypotension shock was considered inconsistent with Starling's law. This prompted an investigation on the hydrodynamics of a porous orifice (G) tube, akin to capillary, and comparing it with Poiseuille's tube. Starling based his hypothesis on Poiseuille's work and considered fluid filtration occurs because of the arterial pressure inside the tube. The arterial pressure inside the $G$ tube induces a negative side pressure on its wall that causes fluid suction and its dynamics is totally different to Poiseuille's tube.

This side pressure of the $G$ tube creates a net negative pressure in a surrounding chamber $\mathrm{C}$ and mixing circulation between fluids inside the $\mathrm{G}$ tube and surrounding it in $\mathrm{C}$. The porous orifice $\mathrm{G}$ tube is based on the ultra-structure of the capillary that has a pre-capillary sphincter and porous slits in its wall that allow the passage of plasma protein- thus no osmotic absorption force exist. The physiological evidence further demonstrated that the capillary works as G tube when fluid flows through the artery and works as Poiseuille's tube when fluid flows through the vein.

On the clinical front, a case series of 23 patients who developed the TURP syndrome of whom the first 3 were mistaken for recognised shock and treated with volume expansion and died. The remaining 20 patients were diagnosed to have VOS and treated with HST all survived. This established the diagnosis of two new types of shock and its treatment. The prospective investigation on 100 consecutive TURP patients of whom 10 cases developed the TURP syndrome further affirmed the discovery of VOS and the effectiveness of HST. This concluded the investigation that is reported here.

\section{Conclusion}

The presented evidence summarises the complete evidence on VOS causing the TURP syndrome and acute dilution hyponataemia both at the clinical and experimental levels. Mistaking VOS for one of the recognised shocks and treating it with further volume expansion is lethal. While HST of $5 \% \mathrm{NaCl}$ or $8.4 \% \mathrm{NaCo}_{3}$ is a life saving treatment for VOS. Starling's law for the capillary-interstitial fluid transfer is proved wrong and an alternative mechanism based on the hydrodynamics of porous orifice $(G)$ tube is provided.

\section{Competing interest: none declared.}

\section{References}

1. Ghanem AN, Ward JP (1990) Osmotic and metabolic sequelae of volumetric overload in relation to the TURP syndrome. Br J Urol 66(1): 71-78.

2. Harrison III RH, Boren JS, Robinson JR (1956) Dilutional hyponatraemic shock: another concept of the transurethral prostatic reaction. J Uro 75(1): 95-110.

3. Arieff AI (1986) Hyponatraemia, convulsion, respiratory arrest and permanent brain damage after elective surgery in healthy women. $\mathrm{N}$
Engl J Med 314(24): 1529-1534.

4. Ashbaugh DG, Bigelow DB, Petty TL, Levine BE (1967) Acute respiratory distress in adults. Lancet 2(7511): 319-323.

5. Danowski TS, Winkler AW, Elkington JR (1946) The treatment of shock due to salt depression; comparison of isotonic, of hypertonic saline and of isotonic glucose solutions. J Clin Invest 25(1): 130-138.

6. Creevy CD (1947) Haemolytic reactions during transurethral prostatic resection. J Uro 58(2): 125-131.

7. Arieff AI, Ayus JC (1993) Endometrial ablation complicated by fatal hyponatraemic encephalopathy. JAMA 270(10): 1230-1232.

8. Istre O, Bjoennes J, Naes R (1994) Postoperative cerebral oedema after Transcervical Endometrial Resection and Uterine Irrigation with 1.5\% Glycine. Lancet 344(8931): 1187-1189.

9. Henderson DJ and Middleton RG (1980) Coma from hyponatraemia of the transurethral resection of prostate. Urology 15(3): 267-271.

10. Bertrand J, Gambini A, Cazalaa JB (1981) Le syndrome de resection de la prostate (TURP) syndrome, mythe oy realite? Jour d' Urologie 87: $1-4$.

11. Bird D, Slade N, Feneley RC (1982) Intravascular complication of transurethral prostatectomy. Br J Uro 54(5): 564-569.

12. Friedman NJ, Hoag MS, Robinson AJ, Aggeler PM (1969) Haemorrhagic syndromes following transurethral resection for benign adenoma. Arch Intern Med 124(3): 341-349.

13. Ekengren J, Hahn RG (1993) Blood loss during transurethral resection of the prostate as measured by the Hemocue photometer. Scand J Uro Nephrol 27(4): 501-507.

14. Evans JW, Singer M, Chapple CR, McCartney N, Walker JM, et al. (1992) Haemodynamic evidence for cardiac stress during transurethral surgery BMJ 304(6828): 666-671.

15. Charlton AJ (1980) Cardiac arrest during transurethral prostatectomy surgery after absorption of 1.5\% glycine. Anaesthesia 35: 804-807.

16. Desmond J (1970) Serum osmolality and plasma electrolytes in patients who develop dilutional hyponatraemia during transurethral resection. Can Jour Surg 13(2): 116-121.

17. Beirne GN, Madsen PO, Burns RO (1965) Serum electrolyte and osmolality changes following transurethral resection of the prostate. J Urol 93: 83-86.

18. Berg G, Fedor EJ, Fisher B (1962) Physiologic observations related to the transurethral resection reaction. The Journal of Urology 87(4): 596-600.

19. Jacobson J (1965) Prolonged respiratory inadequacy following Transurethral Resection of the Prostate. Anaesthesia 20(3): 329-333.

20. Kay MC, Kay J, Begun F, Yeung JE (1985) Vision loss following transurethral resection of the prostate. J Clin Neuroophthalmol 5(4): 273-276.

21. Lessells AM, Honan RP, Haboubi NY, Ali HH, Greene MJ (1982) Death during prostatectomy. J Clin Path 35(1): 117.

22. Hoekstra Pt, Kahnoski R, McCamish MA, Bergen W, Heetderks DR (1983) Transurethral prostatic resection syndrome- a new perspective: Encephalopathy with associated hyperammonaemia. J Uro 130(4): 704-707.

23. Kirshenbaum MA (1979) Sever mannitol induced hyponatraemia complicating transurethral prostatic resection J Uro 121(5): 686-688.

24. Hahn RG (1990) Fluid and electrolyte dynamics during development of the TURP syndrome. Br J Urol 66(1): 79-84.

25. Hahn GH, Zhang W, Rajs J (1996) Pathology of the heart after over hydration with glycine solution in the mouse. APMIS 104(12): 915- 
920

26. Hahn RG, Nennesmo I, Rajs J (1996) Morphological and X-ray Microanalytical Changes in Mammalian Tissue after over hydration with Irrigating Fluids. European Urology 29(3): 355-361.

27. Hahn RG, Nilsson A, Hjelmqvist H, Zhang W, Rundgren M (1996) Renal function during intravenous infusion of urological irrigating fluids in the sheep. Acta Anaesthiol Scand 40(6): 671-678.

28. Hahn RG, Sahdfeldt L, Nymen (1998) Double blind randomized study of symptoms associated with absorption of glycine $1.5 \%$ or mannitol $3 \%$ during transurethral resection of the prostate. J Uro 160(2): 397401.

29. Ghanem AN, Wojtlewski JA, Penney MD (1987) Dangers in treating hyponatraemia. Br Med J (Clin Res Ed) 294(6575): 837.

30. Ghanem AN (2001) Magnetic field-like fluid circulation of a porous orifice tube and its relevance to the capillary-interstitial fluid circulation: preliminary report. Medical Hypotheses 56(3): 325-334.

31. Ghanem AN, Ghanem SA (2016) Volumetric Overload Shocks: Why Is Starling's Law for Capillary Interstitial Fluid Transfer Wrong? The
Hydrodynamics of a Porous Orifice Tube as Alternative. SS Journal $7(6): 245-249$.

32. Pindoria Nisha, Ghanem Salma A, Ghanem Khalid A, Ghanem Ahmed $\mathrm{N}$ (2017) Volumetric overload shocks in the pathoetiology of the transurethral resection prostatectomy syndrome and acute dilution hyponatraemia. Integrative Molecular Medicine 4(2): 1-5.

33. Ghanem Khaled A, Ghanem Ahmed N (2017) Volumetric overload shocks in the patho-etiology of the transurethral resection prostatectomy syndrome and acute dilution hyponatraemia: The clinical evidence based on 23 case series. Basic Research Journal of Medicine and Clinical Sciences 6(4): 36-43.

34. Salma A Ghanem, Khalid A Ghanem, Ghanem AN (2017) Volumetric Overload Shocks in the Patho-Etiology of the Transurethral Resection of the Prostate (TURP) Syndrome and Acute Dilution Hyponatraemia: The Clinical Evidence Based on Prospective Clinical Study of 100 Consecutive TURP Patients. Surg Med Open Access J 1(1): 1-8.

35. Guyton A C, Coleman T G (1968) Regulation of interstitial fluid volume and pressure. Annals New York Academy of Sciences 150: 537-547.

\section{Your next submission with Juniper Publishers will reach you the below assets}

- Quality Editorial service

- Swift Peer Review

- Reprints availability

- E-prints Service

- Manuscript Podcast for convenient understanding

- Global attainment for your research

- Manuscript accessibility in different formats

( Pdf, E-pub, Full Text, Audio)

- Unceasing customer service

Track the below URL for one-step submission https://juniperpublishers.com/online-submission.php 